\title{
Toxic megacolon complicating chemotherapy for acute myeloid leukaemia
}

\author{
M.A. Wodziński, J.A. Snowden and J.T. Reilly \\ Department of Haematology, Northern General Hospital, Sheffield S5 7AU, UK
}

\begin{abstract}
Summary: A 43 year old woman in remission from acute myeloid leukaemia developed abdominal pain, severe melaena, diarrhoea and gram-negative septicaemia whilst severely pancytopenic following consolidation chemotherapy. Subsequently, serial abdominal X-rays showed a progressive toxic megacolon. Conservative management was attempted but, because of radiological evidence of increasing colonic dilatation and incipient perforation, an emergency defunctioning colostomy was performed. The patient recovered and 2 months later the caecostomy was reversed and a right hemicolectomy performed.

This first described case of toxic megacolon following leukaemia treatment is compared with three previously described cases following cytotoxic chemotherapy for other conditions.
\end{abstract}

\section{Introduction}

Toxic megacolon is a life-threatening condition that can develop in any patient with a severe colitis regardless of aetiology. ${ }^{1}$ It is a well-recognized complication of inflammatory bowel disease, ischaemic colitis ${ }^{2}$ and infectious colitis. ${ }^{3}$ Signs of developing toxic dilatation are tachycardia, fever, abdominal distension and tenderness ${ }^{1}$ and a colonic diameter of greater than $7 \mathrm{~cm}$ on abdominal $\mathrm{X}$-ray. ${ }^{2}$

Typhlitis (or 'ileocaecal syndrome') is a serious complication of neutropenic phase of leukaemia treatment characterized by inflammation of the terminal ileum, caecum, appendix and ascending colon associated with the symptom complex of high fever and right-sided abdominal pain with evidence of peritoneal irritation and often diarrhoea and melaena. ${ }^{4}$ In a series of 104 adults with acute myeloid leukaemia, five developed acute abdominal pain requiring emergency surgery comprising two appendicectomies and three right hemicolectomies for typhlitis. ${ }^{5}$

Toxic megacolon has been described following chemotherapy for solid tumours ${ }^{6,7}$ and for psoriasis. ${ }^{8}$ We report a patient with acute myeloid leukaemia who developed typhlitis during the pancytopenic phase following intensive consolidation chemotherapy, which was subsequently complicated by toxic megacolon. This is the first report of toxic megacolon developing after anti-leukaemic chemotherapy.

Correspondence: M.A. Wodziński, M.R.C.P.(UK), M.R.C.Path.

Present address: Department of Haematology, Royal Hospital, Chesterfield S44 5BL, UK.

Accepted: 18 April 1994

\section{Case report}

A 43 year old lady presented with a 2 week history of malaise, intermittent central chest pain, dry cough and drenching night sweats. A blood count showed a haemoglobin of $9.8 \mathrm{~g} / \mathrm{dl}$, white cell count $2.5 \times 10^{9} / 1$ (blasts, some with Auer rods $1.4 \times 10^{9} /$ 1), platelets $320 \times 10^{9} / 1$. The diagnosis of acute myeloid leukaemia $\mathrm{FAB}^{9}$ type $\mathrm{M} 1$ was confirmed by bone marrow aspirate cytology and immunophenotype. Cytogenetic analysis was $46, X X, N A D$.

She was treated with chemotherapy according to the Medical Research Council's AML 10 protocol and achieved complete remission after one course of daunorubicin, cytarabine and etoposide. She received a course of consolidation therapy consisting of daunorubicin, cytarabine and etoposide, and then a course of MACE (amsacrine $100 \mathrm{mg} / \mathrm{m}^{2}$ i.v./day, cytarabine $100 \mathrm{mg} / \mathrm{m}^{2}$ i.v. twice daily and etoposide $100 \mathrm{mg} / \mathrm{m}^{2}$ i.v./day, for 5 days).

On the tenth day after MACE whilst severely pancytopenic (neutrophils $0.1 \times 10^{9} / 1$, platelets $5 \times 10^{9} / 1$ ) she developed an episode of melaena and a small haematemesis. Gastroscopy showed multiple small erosions. Omeprazole and misoprostol were commenced and the bleeding stopped following 15 units of red cells and multiple platelet transfusions, which were HLA matched in view of lymphocytotoxic antibodies to $94 \%$ panel cells. She subsequently developed fever, which responded to intravenous antibiotics, abdominal pain requiring opiate analgesia and diarrhoea, which settled without specific therapy. Blood cultures grew Escherichia coli and Klebsiella aerogenes. Viral titres including cytomegalovirus and Herpes simplex were negative. Stool cultures were negative 
and Clostridium difficile toxins were not found, repeat samples 3 days later were also negative and toxins were not found. Two days later she developed a markedly tender, tense, distended abdomen and abdominal X-ray showed marked colonic distension to $10 \mathrm{~cm}$ with thickening of the bowel wall. She continued to require opiate analgesia but, despite bowel rest, broad-spectrum antibiotics and platelet support, the distension progressed over the ensuing 24 hours with radiological evidence of increasing colonic dilatation with some gas in the bowel wall. Colonoscopic decompression was attempted but was unsuccessful. A urograffin enema showed obstruction to flow of contrast at the level of the mid-transverse colon.

On the 20th day after MACE chemotherapy whilst severely thrombocytopenic but with a recovering neutrophil count, a defunctioning caecostomy was performed with HLA-matched platelet support. Biopsy was considered too hazardous as the colon was friable and haemorrhagic. She made an uneventful recovery. Two months later the caecostomy was reversed electively and a right hemicolectomy was performed as a thickened lesion in the mid-transverse colon was found at laparotomy. Histology of this thickened area showed expansion of the submucosa by fibrosis and a patchy chronic inflammatory cell infiltrate, consistent with a localized area of regeneration following an episode of haemorrhage or ischaemia. There was no evidence of inflammatory bowel disease, neoplasia or residual leukaemia.

The leukaemia relapsed 21 months after initial diagnosis and she received a further course of intensive chemotherapy (DAT3 +10$)$. She had no significant bowel symptoms in the ensuing pancytopenic phase. However, the leukaemia proved to be resistant and she died one month later.

\section{Discussion}

Toxic megacolon has been previously described following chemotherapy. A woman with breast carcinoma treated with epirubicin, 5-fluoruracil and cyclophosphamide developed a toxic megacolon one month after chemotherapy whilst mildly leucopenic. Serial abdominal radiographs showed intraperitoneal free gas and a subtotal colectomy was performed. ${ }^{6} \mathrm{~A}$ case of Hodgkin's disease developed pseudomembranous colitis with positive Clostridium difficile titres in the neutropenic phase following ChlVPP combination chemotherapy. She subsequently developed a toxic megacolon associated with septic shock and an emergency total abdominal colectomy was performed. ${ }^{7}$ A patient with psoriasis became neutropenic after methotrexate therapy and developed toxic megacolon, which eventually resolved with conservative measures. ${ }^{8}$ To our knowledge, toxic megacolon has not been previously described following chemotherapy for the treatment of leukaemia.

The current case had a symptom complex, which developed in the neutropenic phase following intensive anti-leukaemic consolidation chemotherapy, consisting of abdominal pain, fever and rectal bleeding, suggestive of typhlitis., ${ }^{4,10,11}$ Conservative treatment was attempted with blood and platelet transfusion, and antibiotic support. However, over the ensuing days she developed a toxic megacolon associated with a pseudo-obstruction at the mid-transverse colon. There was progressive abdominal distension with radiographic evidence of increasing colonic dilatation with gas in the bowel wall. Because perforation was considered likely, a defunctioning caecostomy was performed, despite the grave risks of surgery in an ill, severely thrombocytopenic haemorrhagic patient requiring HLA-matched platelet support. Biopsies could not be taken at the time because of the friable and haemorrhagic nature of the bowel involved. However, an elective colectomy was performed 2 months later and histology showed no evidence of inflammatory bowel disease, neoplasia or leuko aemia. Infective diarrhoea is unlikely in view negative stool cultures, although culture-negative self-limited colitis ${ }^{3}$ cannot be excluded. Chemo therapy-induced damage to the bowel wall is likely, possibly by ischaemic injury as has been described in typhlitis. ${ }^{12}$ The consequent disturbance of bowel motility may have been compounded by opiate analgesia.

With the limited data available, we suggest the following management of toxic megacolon following chemotherapy for acute myeloid leukaemia Suspected cases should be managed conservatively if possible and be given bowel rest and platelet and antibiotic support as required, and anti-diarrhoeals or opiates stopped if possible. Abdominal X-rays should be performed at least daily to assess progression and to exclude perforation. Endoscopic decompression as explored by Riedler et al.$^{13}$ may be attempted, although in our case this was unsuccessful. Should there be any significant progression such that there is danger of perforation, or if there are any features of the abdominal $X$-ray suggestive of perforation, then emergency surgery must be considered even if the patient is septic, haemorrhagic, neutropenic and thrombocytopenic. A defunctioning colostomy may suffice in most cases as the bowel pathology is reversible, unlike inflammatory bowel disease where total colectomy is often justified. Definitive surgery can be delayed if necessary until the patient has recovered from the pancytopenic phase. 


\section{References}

1. Pettit, S. \& Irving, M.H. Non-specific inflammatory bowel disease. Br Med J 1992, 304: 1367-1371.

2. Heppell, J., Farkouh, E., Dubé, S., Péloquin, A., Morgan, S. $\&$ Bernard, D. Toxic megacolon. An analysis of 70 cases. Dis Colon Rectum 1986, 29: 789-792.

3. Snowden, J.A., Young, M.J. \& McKendrick, M.W. Medical management of toxic dilatation of the colon complicating self-limited colitis - an analysis of nineteen cases. $Q \mathrm{~J} \mathrm{Med}$ 1994, 87: 55-62.

4. Kunkel, J.M. \& Rosenthal, D. Management of the ileocecal syndrome: neutropenic enterocolitis. Dis Colon Rectum 1986, 29: 196-199.

5. Martell, R.W. \& Jacobs, P. Surgery for the acute abdomen in adults with leukaemia. Postgrad Med J 1986, 62: 915-918.

6. De Gara, C.J., Gagic, N., Arnold, A. \& Seaton, T. Toxic megacolon associated with anticancer chemotherapy. Can J Surg 1991, 34: 339-341.

7. Velanovich, V., LaPorta, A.J., Garrett, W.L., Richards, T.B. \& Cornett, P.A. Pseudomembranous colitis leading to toxic megacolon associated with antineoplastic chemotherapy. Report of a case and review of the literature. Dis Colon Rectum 1992, 35: 369-372.
8. Atherton, L.D., Leib, E.S. \& Kaye, M.D. Toxic megacolon associated with methotrexate therapy. Gastroenterology 1984, 86: $1538-1583$.

9. Bennett, J.M., Catovsky, D., Daniel, M.T. et al. Proposed revised criteria for the classification of acute myeloid leukemia. A report of the French-American-British Cooperative Group. Ann Intern Med 1985, 103: 626-629.

10. Hiruki, T., Fernandes, B., Ramsay, J. \& Rother, I. Acute typhlitis in an immunocompromised host. Report of an unusual case and review of the literature. Dig Dis Sci 1992, 37: 1292-1296.

11. Shamberger, R.C., Weinstein, H.J., Delorey, M.J. \& Levey, R.H. The medical and surgical management of typhlitis in children with acute nonlymphocytic (myelogenous) leukemia. Cancer 1986, 57: 603-609.

12. Hammerstrøm, J. The ileocecal syndrome in acute leukaemia - is thrombosis an important part of pathogenesis? Eur $J$ Haematol 1993, 51: 184-185.

13. Riedler, L., Wohlgenannt, D., Stoss, F., Thaler, W. \& Schmitt, K.W. Endoscopic decompression in 'toxic megacolon'. Surg Endosc 1989, 3: 51-53. 